\title{
GENERALIZED MODULE EXTENSION BANACH ALGEBRAS: DERIVATIONS AND WEAK AMENABILITY
}

\author{
MOHAMMAD RAMEZANPOUR,${ }^{1 *}$ and SEDDIGHEH BAROOTKOOB ${ }^{2}$
}

\begin{abstract}
Let $A$ and $X$ be Banach algebras and let $X$ be an algebraic Banach $A$-module. Then the $\ell^{1}$-direct sum $A \times X$ equipped with the multiplication

$$
(a, x)(b, y)=(a b, a y+x b+x y) \quad(a, b \in A, x, y \in X)
$$

is a Banach algebra, denoted by $A \bowtie X$, which will be called " a generalized module extension Banach algebra". Module extension algebras, Lau product and also the direct sum of Banach algebras are the main examples satisfying this framework. We characterize the structure of $n$-dual valued $(n \in \mathbb{N}))$ derivations on $A \bowtie X$ from which we investigate the $n$-weak amenability for the algebra $A \bowtie X$. We apply the results and the techniques of proofs for presenting some older results with simple direct proofs.
\end{abstract}

\section{Introduction And some Preliminaries}

A derivation from a Banach algebra $A$ to a Banach $A$-module $X$ is a bounded linear mapping $D: A \rightarrow X$ such that $D(a b)=D(a) b+a D(b)$ for all $a, b \in A$. For each $x \in X$ the mapping $d_{x}: a \rightarrow a x-x a,(a \in A)$ is a derivation, called the inner derivation implemented by $x$. The concept of $n$-weak amenability was introduced and intensively studied by Dales, Ghahramani and Grønbæk [6]. A Banach algebra $A$ is said to be $n$-weakly amenable $(n \in \mathbb{N})$ if every derivation from $\mathcal{A}$ into $A^{(n)}$ is inner, where $A^{(n)}$ is the $n^{\text {th }}$-dual of $A$, we also write $A^{(0)}=A$. 1 -weak amenability is the so-called weak amenability which was first introduced and studied by Bade, Curtis and Dales [1] for commutative Banach algebras and then by Johnson [11] for a general Banach algebra. $C^{*}-$ algebras and the convolution group algebras are the main examples of $n$-weakly amenable Banach algebras (for a proof see [6], [17]). For more background concerning $n$-weak amenability one may refer to the monograph [5].

Let $A$ and $X$ be Banach algebra and let $X$ be a Banach $A$-module. We say that $X$ is an algebraic Banach $A$-module if for every $x, y \in X$ and $a \in A$,

$$
\begin{aligned}
& a(x y)=(a x) y,(x y) a=x(y a), \quad(x a) y=x(a y), \quad \text { and } \\
& \|a x\| \leq\|a\|\|x\|, \quad\|x a\| \leq\|a\|\|x\|
\end{aligned}
$$

Date: ${ }^{*}$ Corresponding author.

2010 Mathematics Subject Classification. Primary 46H20; Secondary 46H25.

Key words and phrases. Banach algebra, Derivation, $n$-weak amenability. 
Then a direct verification shows that the $\ell^{1}$-direct sum $A \bowtie X$ under the multiplication

$$
(a, x)(b, y)=(a b, a y+x b+x y) \quad(a, b \in A, x, y \in X)
$$

is a Banach algebra which is called the generalized module extension of $A$ by $X$ and denoted by $A \bowtie X$.

It is easy to check that $A \times\{0\}$ is a closed subalgebra while $\{0\} \times X$ is a closed ideal of $A \bowtie X$, and that $(A \bowtie X) /(\{0\} \times X)$ is isometrically isomorphic to $A \times\{0\}$.

The main examples of generalized module extensions are listed as follows:

- (The classical module extension algebras) The (classical) module extension algebra $A \ltimes X$, as introduced in [2], is the $\ell^{1}$-direct sum of $A$ by the Banach $A$-module $X$ equipped with the multiplication

$$
(a, x)(b, y)=(a b, a y+x b) \quad(a, b \in A, x, y \in X) .
$$

Clearly, $A \ltimes X$ is a generalized module extension Banach algebra, in which $X$ is equipped with the trivial product $x y=0$. Module extension Banach algebras are known as a rich source of (counter-)examples in various situations in abstract harmonic analysis and functional analysis. Some aspects of the Banach algebra $A \ltimes X$ have been described in [2]. In [16] Zhang has characterized the structures of derivations from $A \ltimes X$ into various duals from which he investigated the $n$-weak amenability properties of $A \ltimes X$. The class of module extension Banach algebras also includes the triangular Banach algebra whose weak amenability has been investigated in [9].

- $(\theta$-Lau product of Banach algebras $)$ Let $A$ and $B$ be Banach algebras and let $\theta \in \sigma(A)$. Then the $\theta$-Lau product $A_{\theta} \times B$ which is equipped with the multiplication

$$
(a, b)(c, d)=(a c, \theta(a) d+\theta(c) b+b d) \quad(a, c \in A, b, d \in B),
$$

can be viewed as a generalized module extension algebra, in which $B$ endowed with the module operations $a b=b a=\theta(a) b$ is an algebraic Banach $A$-module. This product was introduced by Lau [13] for certain class of Banach algebras and followed by Sangani Monfared [15] for the general case. A very familiar example is the case that $A=\mathbb{C}$ with $\theta=\iota$ as the identity character, that we get the unitization $B^{\sharp}=\mathbb{C}{ }_{\iota} \times B$ of $B$. Some aspects of $A_{\theta} \times B$ are investigated in $[7,8,12]$. In particular, the $n$-weak amenability of $A_{\theta} \times B$ are discussed in [8].

- (T-Lau product of Banach algebras) Let $A$ and $B$ be Banach algebras and let $T: A \rightarrow B$ be a continuous homomorphism with $\|T\| \leq 1$. Then the $T$-Lau product $A_{T} \times B$ which is equipped with the multiplication

$$
(a, b)(c, d)=(a c, T(a) d+b T(c)+b d) \quad(a, c \in A, b, d \in B),
$$

can also be viewed as a generalized module extension algebra, in which $B$ furnished with the module operations $a b=T(a) b$ and $b a=b T(a)$ is an algebraic Banach $A$-module. This type of product was first introduced by 
Bhatt and Dabhi [3] for the case where $B$ is commutative and was extended by Javanshiri and Nemati for the general case [10]; see also [14].

In particular, in the case $T=0$, we arrive at the $\ell^{1}$-direct sum $A \oplus_{1} B$ of two Banach algebra $A$ and $B$, equipped with the pointwise multiplication

$$
(a, b)(c, d)=(a b, c d) \quad(a, c \in A, b, d \in B) .
$$

Recently, Choi [4] has demonstrated that the $T$-Lau product $A_{T} \times B$ is isomorphic as a Banach algebra to the usual direct sum $A \oplus_{1} B$.

For a generalized module extension Banach algebra $A \bowtie X$ one can directly checked that the $n^{\text {th }}$-dual $(A \bowtie X)^{n}$ as a Banach $(A \bowtie X)$-module enjoys the following module operations:

$$
\begin{aligned}
(F, G)(a, x) & =(F a, F x+G a+G x), \\
(a, x)(F, G) & =(a F, x F+a G+x G), \\
(f, g)(a, x) & =(f a+g x, g x+g a), \\
(a, x)(f, g) & =(a f+x g, x g+a g),
\end{aligned}
$$

for all $a \in A, F \in A^{(2 n)}, f \in A^{(2 n+1)}, x \in X, G \in X^{(2 n)}$ and $g \in X^{(2 n+1)}$.

The paper is organized as follows: In Section 2 we investigate $n$-weak amenability of $A \bowtie X$ in the case where $n$ is odd. Then we apply our results for the particular cases, $A \ltimes X, A_{\theta} \times B, A_{T} \times B$ and $A \oplus_{1} B$. Section 3 follows the same discipline for the case $n$ is even. The case where $X$ is unital is invistigated in Section 4. This special case provides some simplifications in the constructions of derivations to various duals of $A \bowtie X$.

\section{2. $(2 n+1)$-WEAK AMENABILITY OF $A \bowtie X$}

In this section we characterize $(2 n+1)$-weak amenability of the generalized module extension Banach algebra $A \bowtie X$ in terms of the $(2 n+1)$-weak amenability of $A$ and $X$. We begin with the following elementary lemma characterizing derivations from $A \bowtie X$ into $(A \bowtie X)^{(2 n+1)}$.

Lemma 2.1. Every derivation $D: A \bowtie X \rightarrow(A \bowtie X)^{(2 n+1)} \quad(n \in \mathbb{N} \cup\{0\})$ enjoys the presentation

$$
D(a, x)=\left(D_{A}(a)+T_{A}(x), D_{X}(a)+T_{X}(x)\right)
$$

where

(a) $D_{A}: A \rightarrow A^{(2 n+1)}$ and $D_{X}: A \rightarrow X^{(2 n+1)}$ are derivations.

(b) $T_{A}: X \rightarrow A^{(2 n+1)}$ is a bounded linear map such that for every $a \in A, x \in X$, $T_{A}(a x)=a T_{A}(x)+D_{X}(a) x$ and $T_{A}(x a)=T_{A}(x) a+x D_{X}(a)$.

(c) $T_{X}: X \rightarrow X^{(2 n+1)}$ is a derivation such that for every $a \in A, x \in X$, $T_{X}(a x)=D_{X}(a) x+a T_{X}(x), T_{X}(x a)=x D_{X}(a)+T_{X}(x) a$ and $T_{X}(x) y+$ $x T_{X}(y)=T_{A}(x y)$.

Moreover, $D$ is inner, that is, $D=d_{(f, g)}$ for some $f \in A^{(2 n+1)}, g \in X^{(2 n+1)}$ if and only if $D_{A}=d_{f}, D_{X}=d_{g}$ and $T_{X}=d_{g}$ are inner derivations and $T_{A}=\delta_{g}$, where $\delta_{g}(x)=x g-g x,(x \in X)$. 
We are ready to prove one of our main result characterizing $(2 n+1)$-amenability of $A \bowtie X$.

Theorem 2.2. A generalized module extension Banach algebra $A \bowtie X$ is $(2 n+$ 1) -weakly amenable if and only if

(1) A is $(2 n+1)$-weakly amenable.

(2) If $T_{X}: X \rightarrow X^{(2 n+1)}$ is a derivation such that there exist a derivation $D_{X}: A \rightarrow X^{(2 n+1)}$ and a bounded linear map $T_{A}: X \rightarrow A^{(2 n+1)}$ satisfy$\operatorname{ing} T_{A}(a x)=a T_{A}(x)+D_{X}(a) x, T_{A}(x a)=T_{A}(x) a+x D_{X}(a), T_{X}(a x)=$ $D_{X}(a) x+a T_{X}(x), T_{X}(x a)=x D_{X}(a)+T_{X}(x) a$ and $T_{X}(x) y+x T_{X}(y)=$ $T_{A}(x y)$, for all $a \in A, x \in X$, then $T_{X}$ is inner.

(3) If $D_{X}: A \rightarrow X^{(2 n+1)}$ is a derivation such that $x D_{X}(a)=D_{X}(a) x=0$, for all $a \in A, x \in X$, then there exists an element $g \in X^{(2 n+1)}$ such that $D_{X}=d_{g}$ and $x g=g x$, for all $x \in X$.

(4) If $T_{A}: X \rightarrow A^{(2 n+1)}$ is a bounded $A$-module homomorphism such that $T_{A}(x y)=0$ for all $x, y \in X$, then $T_{A}=0$.

As a consequence we have the following result which extends [8, Theorem 2.4].

Proposition 2.3. Suppose that both $A$ and $X$ are $(2 n+1)$-weakly amenable. Then $A \bowtie X$ is $(2 n+1)$-weakly amenable when either of the following condition holds.

(1) $\left\langle X X^{(2 n)}+X^{(2 n)} X\right\rangle$ is dense in $X^{(2 n)}$.

(2) $\left\langle X A^{(2 n)}+A^{(2 n)} X\right\rangle$ is dense in $X^{(2 n)}$.

Applying Theorem 2.2 for the calassical module extension Banach algebra $A \ltimes$ $X$ we arrive at the following result which has already proved by Zhang [16] by a slightly different method.

Corollary 2.4 ([16, Theorem 2.1]). A (classical) module extension Banach algebra $A \ltimes X$ is $(2 n+1)$-weakly amenable if and only if

(1) $A$ is $(2 n+1)$-weakly amenable.

(2) The only $A$-module morphism $T: X \rightarrow X^{(2 n+1)}$ such that $x T(y)+T(x) y=$ 0 , in $A^{(2 n+1)}$, for all $x, y \in X$, is zero.

(3) $H^{1}\left(A, X^{(2 n+1)}\right)=\{0\}$; that is, every derivation from $A$ to $X^{(2 n+1)}$ is inner.

(4) For every continuous $A$-module morphism $S: X \rightarrow A^{(2 n+1)}$, there exists $f \in X^{(2 n+1)}$ such that $a f=f a$ for all $a \in A$ and $S(x)=x f-f x$ for all $x \in X$.

Proof. It is enough to use Theorem 2.2 for the case where $x y=0$ for all $x, y \in$ $X$.

As another consequence of Theorem 2.2, we use it for the $\theta$-Lau product Banach algebra $A_{\theta} \times B$. Then we get the following characterization for $(2 n+$ 1)-weak amenability of $A_{\theta} \times B$ which extends the related results in [8].

Corollary 2.5. The $\theta$-Lau product Banach algebra $A_{\theta} \times B$ is $(2 n+1)$-weakly amenable if and only if

(1) $A$ is $(2 n+1)$-weakly amenable. 
(2) Every derivation $T: B \rightarrow B^{(2 n+1)}$ for which there exists a bounded $A$-module homomorphism $S: B \rightarrow A^{(2 n+1)}$ such that $(T(b)(d)+T(d)(d)) \theta=S(b d)$ for all $b, d \in B$ is inner.

(3) The only bounded linear map $D: A \rightarrow B^{(2 n+1)}$ such that $D(a c)=\theta(a) D(c)+$ $\theta(c) D(a)$ and $b D(a)=0=D(a) b$ for all $a, c \in A$ and $b \in B$, is zero.

(4) The only bounded linear operator $S: B \rightarrow A^{(2 n+1)}$ such that $S(b d)=$ $0, a S(b)=S(b) a=\theta(a) S(b)$ for all $a \in A$ and $b, d \in B$, is zero.

Applying Theorem 2.2 for the case where $X=A$, with the multiplication as the module operation, we have the following characterization of $(2 n+1)$-weak amenability of $A \bowtie A$.

Corollary 2.6. $A \bowtie A$ is $(2 n+1)$-weakly amenable if and only if $A$ is $(2 n+$ 1)-weakly amenable.

Applying Theorem 2.2 for the direct sum algebra $A \oplus_{1} B$ we get the following well known result.

Corollary 2.7. $A \oplus_{1} B$ is $(2 n+1)$-weakly amenable if and only if both $A$ and $B$ are $(2 n+1)$-weakly amenable.

\section{3. (2n)-WEAK AMENABILITY OF $A \bowtie X$}

In this section we characterize $(2 n)$-weak amenability of the generalized module extension Banach algebra $A \bowtie X$ in terms of the $(2 n)$-weak amenability of $A$ and $X$. Similar to the previous section (Lemma 2.1) we begin with the following elementary lemma characterizing the derivations from $A \bowtie X$ into $(A \bowtie X)^{(2 n)}$.

Lemma 3.1. Every derivation $D: A \bowtie X \rightarrow(A \bowtie X)^{(2 n)}, \quad(n \in \mathbb{N} \cup\{0\})$, enjoys the presentation

$$
D(a, x)=\left(D_{A}(a)+T_{A}(x), D_{X}(a)+T_{X}(x)\right)
$$

where

(a) $D_{A}: A \rightarrow A^{(2 n)}$ and $D_{X}: A \rightarrow X^{(2 n)}$ are derivation.

(b) $T_{A}: X \rightarrow A^{(2 n)}$ is a bounded $A$-module homomorphism satisfying $T_{A}(x y)=$ 0 , for all $x, y \in X$.

(c) $T_{X}: X \rightarrow X^{(2 n)}$ is a bounded linear map such that $T_{X}($ ax $)=D_{A}(a) x+$ $D_{X}(a) x+a T_{X}(x), T_{X}(x a)=x D_{A}(a)+x D_{X}(a)+T_{X}(x) a$ and $T_{X}(x y)=$ $x T_{X}(y)+T_{X}(x) y+x T_{A}(y)+T_{A}(x) y$, for each $a \in A, x, y \in X$.

Moreover, $D=d_{(F, G)}$ for some $F \in A^{(2 n)}, G \in X^{2 n}$, if and only if $D_{A}=d_{F}, D_{X}=$ $d_{G}$ and $T_{X}=d_{G}+\delta_{F}$ (are inner derivations), and $T_{A}=0$, where $\delta_{F}(x)=$ $x F-F x,(x \in X)$.

The next result is the $(2 n)$-version of Theorem 2.2.

Theorem 3.2. A generalized module extension Banach algebra $A \bowtie X$ is $(2 n)$-weakly amenable if and only if

(1) If $D_{A}: A \rightarrow A^{(2 n)}$ is a derivation such that there exist a derivation $D_{X}$ : $A \rightarrow X^{(2 n)}$, a bounded linear operator $T_{X}: X \rightarrow X^{(2 n)}$ and a bounded $A$ module homomorphism $T_{A}: X \rightarrow A^{(2 n)}$ satisfying $T_{A}(x y)=0, T_{X}(a x)=$ 
$D_{A}(a) x+D_{X}(a) x+a T_{X}(x), T_{X}(x a)=x D_{A}(a)+x D_{X}(a)+T_{X}(x) a$ and $T_{X}(x y)=x T_{X}(y)+T_{X}(x) y+T_{A}(x) y+x T_{A}(y)$, for all $a \in A$ and $x, y \in X$, then $D_{A}$ is inner.

(2) If $T_{X}: X \rightarrow X^{(2 n)}$ is a bounded linear operator such that there exist a derivation $D_{X}: A \rightarrow X^{(2 n)}$ and a bounded A-module homomorphism $T_{A}:$ $X \rightarrow A^{(2 n)}$ satisfying $T_{A}(x y)=0, T_{X}(a x)=D_{X}(a) x+a T_{X}(x), T_{X}(x a)=$ $x D_{X}(a)+T_{X}(x) a$ and $T_{X}(x y)=x T_{X}(y)+T_{X}(x) y+T_{A}(x) y+x T_{A}(y)$, for all $a \in A$ and $x, y \in X$, then there exist elements $F \in A^{(2 n)}$ and $G \in X^{(2 n)}$ such that $T_{X}=\delta_{F}+d_{G}$ and $a F=F a$ for all $a \in A$.

(3) If $D_{X}: A \rightarrow X^{(2 n)}$ is a derivation such that $x D_{X}(a)=D_{X}(a) x=0$, in $X^{(2 n)}$, for all $a \in A$ and $x \in X$, then there exist elements $G \in X^{(2 n)}$ and $F \in A^{(2 n)}$ such that $D_{X}=d_{G}, d_{G}+\delta_{F}=0$ and $a F=$ Fa for all $a \in A$.

(4) If $T_{A}: X \rightarrow A^{(2 n)}$ is a bounded $A$-module homomorphism such that $T_{A}(x y)=$ 0 for all $x, y \in X$, and there exists a bounded A-module homomorphism $T_{X}: X \rightarrow X^{(2 n)}$ satisfying $T_{X}(x y)=x T_{X}(y)+T_{X}(x) y+T_{A}(x) y+x T_{A}(y)$, for all $x, y \in X$, then $T_{A}=0$.

As an application of the latter Theorem we bring the following even analogue of Proposition 2.3.

Proposition 3.3. Suppose that $X^{2}$ is dense in $X$ and both $A$ and $X$ are $(2 n)$-weakly amenable. If $\left\langle X X^{(2 n-1)}+X^{(2 n-1)} X\right\rangle$ is dense in $X^{(2 n-1)}$, then $A \bowtie X$ is $(2 n)$-weakly amenable.

We apply Theorem 3.2 for a classical generalized module extension Banach algebra $A \ltimes X$ to obtain the following result of Zhang [16].

Corollary 3.4 ([16, Theorem 2.2]). A (classical) module extension Banach algebra $A \ltimes X$ is $(2 n)$-weakly amenable if and only if the following conditions hold.

(1) The only derivation $D: A \rightarrow A^{(2 n)}$ for which there exists a continuous operator $T: X \rightarrow X^{(2 n)}$ such that $T(a x)=a T(x)+D(a) x$ and $T(x a)=$ $T(x) a+x D(a)$ for all $a \in A$ and $x \in X$, are inner derivations.

(2) For every continuous $A$-module morphism $T: X \rightarrow X^{(2 n)}$, there exists a $F \in A^{(2 n)}$ such that $T(x)=x F-F x$ and $a F=F a$ for all $x \in X, a \in A$.

(3) $H^{1}\left(A, X^{(2 n)}\right)=\{0\}$.

(4) The only continuous $A$-module morphism $S: X \rightarrow A^{(2 n)}$ for which $x S(y)+$ $S(x) y=0$, in $X^{(2 n)}$, for all $x, y \in X$, is zero.

Proof. It is enough to use Theorem 3.2 for the case where $x y=0$ for all $x, y \in$ $X$.

Applying Theorem 3.2 for the $\theta$-Lau product Banach algebra $A_{\theta} \times B$, we get the following characterization for $(2 n)$-weak amenability of $A_{\theta} \times B$ which extends the related results in [8].

Corollary 3.5. A $\theta$-Lau product Banach algebra $A_{\theta} \times B$ is (2n)-weakly amenable if and only if the following conitions hold. 
(1) The only derivations $D: A \rightarrow A^{(2 n)}$ for which there is a derivation $D_{1}$ : $A \rightarrow B^{(2 n)}$ such that $D_{1}(a) b=b D_{1}(a)=-D(a)(\theta) b$ for all $a \in A$ and $b \in B$, are inner derivations.

(2) $B$ is $(2 n)$-weakly amenable.

(3) The only bounded linear operator $D_{1}: A \rightarrow B^{(2 n)}$ such that $D(a c)=$ $\theta(a) D(c)+\theta(c) D(a)$ and $b D_{1}(a)=0=D_{1}(a) b$ for all $a, c \in A, b \in B$, is zero.

(4) The only bounded linear operator $S: B \rightarrow A^{(2 n)}$ for which $S(b d)=$ $0, a S(b)=S(b) a=\theta(a) S(b)$ for all $a \in A, b, d \in B$ and there is a bounded linear operator $T: B \rightarrow B^{(2 n)}$ such that $T(b d)=b T(d)+T(b) d+S(b)(\theta) d+$ $S(d)(\theta) b$ for all $b, d \in B$, is zero.

Applying Theorem 3.2 for the case $X=A$, with the multiplication as module operation, we get the following characterization of $(2 n)$-weak amenability of $A \bowtie A$.

Corollary 3.6. $A \bowtie A$ is $(2 n)$-weakly amenable if and only if

(1) $A$ is $(2 n)$-weakly amenable.

(2) the only bounded homomorphism $S: A \rightarrow A^{(2 n)}$ for which $a S(c)=S(a) c=$ $S(a c)=0$ for each $a, c \in A$, is zero.

Applying Theorem 3.2 for the direct sum algebra $A \oplus_{1} B$ we get the following result.

Corollary 3.7. The direct sum $A \oplus_{1} B$ of two Banach algebra $A$ and $B$ is $(2 n)$-weakly amenable if and only if

(1) Both $A$ and $B$ are $(2 n)$-weakly amenable.

(2) The only bounded homomorphism $D: A \rightarrow B^{(2 n)}$ for which $D(a c)=0$ and $b D(a)=D(a) b=0$ for all $a, c \in A, b \in B$, is zero.

(3) The only bounded homomorphism $S: B \rightarrow A^{(2 n)}$ for which $S(b d)=0$ and $a S(b)=D(b) a=0$ for all $a \in A, b, d \in B$, is zero.

If we combine Propositions 2.3 and 3.3, we have the following result providing some sufficient conditions for $n$-weak amenability of $A \bowtie X$. In particular, in the setting of $\theta$-Lau products, it improves [8, Proposition 2.4], as well as, for T-Lau products, it improve [10, Proposition 3.5].

Proposition 3.8. Suppose that $X^{2}$ is dense in $X$ and for some $n>0$ either $\overline{X X^{(n-1)}}=X^{(n-1)}$ or $\overline{X^{(n-1)} X}=X^{(n-1)}$. If $A$ and $X$ are $n$-weakly amenable then $A \bowtie X$ is $n$-weakly amenable.

We recall from [6, Proposition 1.2] that if $A$ is weakly amenable, then $\overline{A A}=A$. Thus as a rapid consequence of Proposition 3.8 we get,

Corollary 3.9. If $A$ and $X$ are weakly amenable then $A \bowtie X$ is weakly amenable.

From Corollaries 2.7, 3.7 and Proposition 3.8 we get the following result.

Corollary 3.10. Suppose that $B^{2}$ is dense in $B$ and for some $n>0$ either $\overline{B B^{(n-1)}}=B^{(n-1)}$ or $\overline{B^{(n-1)} B}=B^{(n-1)}$. Then $A \oplus_{1} B$ is $n$-weakly amenable if and only if both $A$ and $B$ are $n$-weakly amenable. 
From Corollaries 2.6, 3.6 we immediately obtain the next result.

Corollary 3.11. Suppose that $A^{2}$ is dense in $A$ then $A \bowtie A$ is $n$-weakly amenable if and only if $A$ is $n$-weakly amenable.

\section{THE CASE WHERE $X$ IS UNITAL}

In this section we assume that $X$ is unital with the identity $1_{X}$. In this case the characterizations of derivations $D: A \bowtie X \rightarrow(A \bowtie X)^{(n)}$ presented in Lemma 2.1 and 3.1 can be considerably simplified. This result extends [10, Corollareis $3.9,3.10]$ and [8, Proposition 3.1].

Lemma 4.1. Let $X$ be unital with the identity $1_{X}$. Then

(1) $D: A \bowtie X \rightarrow(A \bowtie X)^{(2 n+1)}$ is a derivation if and only if

$D(a, x)=\left(D_{A}(a)+T_{X}(x) 1_{X}, T_{X}\left(a 1_{X}\right)+T_{X}(x)\right), \quad(a \in A, x \in X) ;$ where, $D_{A}: A \rightarrow A^{(2 n+1)}$ and $T_{X}: X \rightarrow X^{(2 n+1)}$ are derivations and $T_{X}\left(1_{X} a\right)=T_{X}\left(a 1_{X}\right)$ for all $a \in A$ and $T_{X}(x) 1_{X}=1_{X} T_{X}(x)$, in $A^{(2 n+1)}$, for all $x \in X$. Moreover, $D=d_{(f, g)}$ is inner derivation if and only if $D_{A}=d_{f}$ and $T_{X}=d_{g}$ are inner derivations.

(2) $D: A \bowtie X \rightarrow(A \bowtie X)^{(2 n)}$ is a derivation if and only if

$D(a, x)=\left(D_{A}(a), T_{X}\left(a 1_{X}\right)-D_{A}(a) 1_{X}+T_{X}(x)\right), \quad(a \in A, x \in X) ;$

where, $D_{A}: A \rightarrow A^{(2 n)}$ and $T_{X}: X \rightarrow X^{(2 n)}$ are derivations, $T_{X}\left(1_{X} a\right)-$ $1_{X} D_{A}(a)=T_{X}\left(a 1_{X}\right)-D_{A}(a) 1_{X}$ for all $a \in A$ and $D(a)=D_{A}(a) 1_{X}$ is a bounded derivation from $A$ into $X^{(2 n)}$. Moreover, $D=d_{(F, G)}$ is inner derivation if and only if $D_{A}=d_{F}$ and $T_{X}=d_{G}+\delta_{F}=d_{G+1_{X} F}$ are inner derivations.

As a consequence of Proposition 3.8 we give the next result concerning to the (n)-weak amenability of $A \bowtie X$ in the case where $X$ is unital. This result covers [8, Theorem 3.1] and [10, Proposition 3.11] in the special case.

Theorem 4.2. Suppose that $X$ is unital.

(1) If $A$ and $X$ are $(2 n+1)$-weakly amenable then $A \bowtie X$ is $(2 n+1)$-weakly amenable.

(2) If $A \bowtie X$ is $(2 n+1)$-weakly amenable then $A$ is $(2 n+1)$-weakly amenable and the only derivations $T_{X}: X \rightarrow X^{(2 n+1)}$ which is also an A-module homomorphism and $T_{X}(x) 1_{X}=1_{X} T_{X}(x)$ are inner derivations.

(3) If $A \bowtie X$ is $(2 n+1)$-weakly amenable then $A$ is $(2 n+1)$-weakly amenable and $X$ is $(2 n+1)$-cyclicly weak $A$-module amenable.

(4) If $A$ and $X$ are $(2 n)$-weakly amenable then $A \bowtie X$ is $(2 n)$-weakly amenable.

Acknowledgments. The authors would like to express their appreciation to Professor H. R. Ebrahimi Vishki for introducing them to the subject of this work and all of his encouragement and valuable comments which provided significant improvements to this article. 


\section{REFERENCES}

[1] W.G. Bade, P.C. Curtis, and H.G. Dales, Amenability and weak amenability for Beurling and Lipschitz algebras, Proc. London Math. Soc. 55 (1987), 359-377.

[2] W.G. Bade, H.G. Dales and Z.A. Lykova, Algebraic and strong splittings of extensions of Banach algebras, Mem. Amer. Math. Soc. 137, (1999).

[3] S.J. Bhatt and P.A. Dabhi, Arens regularity and amenability of Lau product of Banach algebras defined by a Banach algebra morphism, Bull. Austral. Math. Soc. 87, (2013), 195-206.

[4] Y. Choi, Triviality of the generalized Lau product associated to a Banach algebra homomorphism, ArXiv:1511.07868v3, (2016).

[5] H.G. Dales, Banach algebras and automatic countinuity, Clarendon Press, Oxford, (2000).

[6] H.G. Dales, F. Ghahramani, and N. Grønbæk, Derivations into iterated duals of Banach algebras, Studia Math. (1) 128 (1998), 19-54.

[7] H.R. Ebrahimi Vishki and A.R. Khoddami, Character inner amenability of certain Banach algebras, Colloq. Math. 122 (2011), 225-232.

[8] H.R. Ebrahimi Vishki and A.R. Khoddami, n-Weak amenability for Lau product of Banach algebras, Politehn. Univ. Bucharest Sci. Bull. Ser. A Appl. Math. Phys. 77 (2015), 177-184.

[9] B.E. Forrest and L.W. Marcoux, Weak amenability of triangular Banach algebras, Trans. Amer. Math. Soc. 354 (2002), 1435-1452.

[10] H. Javanshiri and M. Nemati, On a certain product of Banach algebras and some of its properties, Proc. Rom. Acad. Ser. A Math. Phys. Tech. Sci. Inf. Sci. 15 (2014), 219-227.

[11] B.E. Johnson, Weak amenability of group algebras, Bull. London Math. Soc. 23 (1991), 281-284.

[12] E. Kaniuth, The Bochner-Schoenberg-Eberline property and spectral synthesis for certain Banch algebra products, Canad. J. Math. 67 (2015), 827-847.

[13] A.T.-M. Lau, Analysis on a class of Banach algebras with applications to harmonic analysis on locally compact groups and semigroups, Fund. Math. 118 (1983), 161-175.

[14] M. Nemati and H. Javanshiri, Some homological and cohomological notions on T-Lau product of Banach algebras, Banach J. Math. Anal. 9 (2015), 183-195.

[15] M. Sangani Monfared, On certain products of Banach algebras with applications to harmonic analysis, Studia Math. 178 (3) (2007), 277-294.

[16] Y. Zhang, Weak amenability of module extensions of Banach algebras, Trans. Amer. Math. Soc. 354 (10) (2002), 4131-4151.

[17] Y. Zhang, 2m-Weak amenability of group algebras, J. Math. Anal. Appl. 396 (2012), $412-416$.

${ }^{1}$ School of Mathematics and Computer Science, Damghan University, P. O. Box 36716, Damghan 41167, Iran.

E-mail address: ramezanpour@du.ac.ir

2 Department of Mathematics, University of Bojnord, P.O. Box 1339, Bojnord, IRAN.

E-mail address: s.barutkub@ub.ac.ir 